Evanthia Monogioudi*, Joanna Sheldon, Pier Luigi Meroni, Dana Petronela Hutu, Heinz Schimmel and Ingrid Zegers, on behalf of the Committee for the Harmonisation of Autoimmune Testing (C-HAT) of the International Federation of Clinical Chemistry and Laboratory Medicine (IFCC)

\title{
Certified reference material against PR3 ANCA IgG autoantibodies. From development to certification
}

https://doi.org/10.1515/cclm-2018-1095

Received October 9, 2018; accepted January 8, 2019; previously published online February 21, 2019

\section{Abstract}

Background: The importance of the standardisation of immunoassays for autoantibodies has been widely discussed. The appropriate use of certified reference materials (CRM) could contribute to a more accurate diagnosis and follow-up of a series of diseases such as small vesselassociated vasculitis. This is a systemic autoimmune disorder during which two autoantibodies can be present, MPO ANCA IgG and PR3 ANCA IgG. Results from different commercially available immunoassays used for PR3 ANCA IgG measurement can vary significantly. Therefore the potential for improvement using a suitable certified reference material was assessed and led to the development of a CRM.

Methods: Thirty clinical samples were evaluated using 10 immunoassays. The correlation between results from these assays was assessed in a pairwise manner. Feasibility studies were conducted in order to find a reference material format most suitable for the preparation of a CRM.

Results: The evaluation of two sets of 30 clinical samples with 10 assays showed that differences between assays can result in different interpretations for individual clinical samples. Most of the samples had the same result classification in all assays. However, six of the samples tested led to inconsistent results.

Conclusions: The correlation between results from clinical samples was systematically good for combinations of

\footnotetext{
*Corresponding author: Evanthia Monogioudi, European Commission, Joint Research Centre, Retieseweg 111, 2440 Geel, Belgium, Phone: +32 14571 906, Fax: +32 14571548 , E-mail: Evanthia.monogioudi@ec.europa.eu. https://orcid.org/0000-0001-8525-8921 Joanna Sheldon: Protein Reference Unit and Immunopathology Department, St Georges' Hospital, London, UK Pier Luigi Meroni: Istituto G. Pini, University of Milan, Department of Clinical Sciences and Community Health and Istituto Auxologico Italiano, Milan, Italy

Dana Petronela Hutu, Heinz Schimmel and Ingrid Zegers: European Commission, Joint Research Centre, Geel, Belgium
}

eight of those assays. Therefore, it should be possible to improve the comparability of results using a commutable CRM for calibration. Based on these studies, a final format for the CRM was selected and eventually produced and certified for its PR3 ANCA IgG content.

Keywords: autoimmune disease; commutability; immunoassay; in vitro diagnostics; PR3 ANCA; reference material; standardisation.

\section{Introduction}

\section{PR3 and disease}

Anti-neutrophil cytoplasmic antibodies (ANCAs) were described as crucial diagnostic markers for small-vessel vasculitis already in 1985 [1]. There are two main targets for the antibodies responsible for most of the sub-categories of small-vessel vasculitis. Antibodies against myeloperoxidase (MPO) are detected mostly in microscopic polyangiitis (MPA) and eosinophilic granulomatosis with polyangiitis (EGPA), and against proteinase 3 (PR3) in granulomatosis with polyangiitis (GPA) [2]. Interestingly, there seems to be a geographical pattern in the appearance of all the types, with PR3 ANCA being mostly encountered in Northern Europe and southern New Zealand, while MPO ANCA is more often encoutered in Mediterranean countries. In addition to the geographical pattern, PR3 ANCA seems to be more prevalent in Caucasians than in Asians or Africans [3]

\section{PR3 measurements}

According to the Chapel Hill Consensus of 2012, the laboratory investigation includes a screening test by indirect immunofluorescence (IIF), which can be followed by confirmation with specific enzyme-linked immunosorbent assays (ELISAs) for IgG antibodies to PR3 and/or MPO [4]. Recently, a group of international experts proposed a revision of the consensus, where the value of immunoassay 
testing for diagnosis is highlighted. Modern immunoassays have improved performance especially over the more traditional IIF [5]. PR3 ANCA IgG antibodies present a wide variation in the concentration values generated for the same sample by different commercially available immunoassays. Part of the reason is that companies use arbitrary units, often different ones, making it impossible to compare results between the various available assays.

\section{PR3 CRM}

In 2010, the International Federation of Clinical Chemistry and Laboratory Medicine (IFCC) formed a working group to collaborate with the Joint Research Centre of the European Commission on the harmonisation of autoimmune antibody testing. The first objective of this group was to establish whether it is possible to develop reference systems including CRMs that have property values metrologically traceable to the International System of Units (SI) for selected autoantibody tests. The ultimate aim was to prepare CRMs that would be widely available for standardisation of serology testing of several autoantibodies, amongst them, a CRM for PR3 ANCA IgG antibodies.

The development of a CRM for PR3 ANCA IgG was the logical continuation after the successful development and release of ERM-DA476/IFCC, a CRM for MPO ANCA IgG. A reference material has to resemble a clinical sample so as to minimise calibration bias and be of value [6-9]. This property of a CRM is known as commutability. It can be simply defined as the property of a reference material (RM) to mimic the characteristics of a typical clinical sample in two or more measurement procedures for a stated measurand [10]. Two commutability studies were performed in a period of 3 years, comparing the results for a representative number of clinical samples and candidate reference materials across various assays. In the first study the comparability of results from a selected number of assays was assessed, and different raw materials and CRM formats were evaluated. A second study was performed in order to further validate the most promising candidate CRMs, using a smaller selection of assays. The measurements for the second study were performed using a different set of clinical samples.

\section{Comparability of results from different assays}

There are a number of factors contributing to the variability of autoantibody results. Monogioudi et al. summarised them in a recent paper [11]. These factors include a wide selection from the principles of in vitro diagnostic (IVD) assays, the repeatability and intermediate precision of each one of them, the different scales and units used from the manufacturers but also the sample-specific variation, mainly due to the specific antigen used for the development of an assay.

For our studies, from their initial steps of the feasibility and commutability to eventually the development of the CRM and its characterisation, most of these factors were taken into account and eventually only the sample specific variation and the different scales used by the different assays were left to account for any differences observed.

In this paper we are mainly presenting the results of the preliminary correlation studies for PR3 ANCA IgG assays, report the results in analytical terms, describe how the CRM was prepared and briefly discuss the characterisation process of this material. Further details and information can be found on the certification report of the material, ERM-DA483/IFCC [12].

\section{Materials and methods}

\section{Serum samples}

Clinical samples were collected based on the amounts available and their PR3 ANCA IgG concentration. Two separate commutability assessments were performed and in total 60 serum samples were used (30 in the first and 30 in the second study). Serum samples were from patients undergoing PR3 ANCA IgG testing at the Protein Reference Unit and Immunopathology Department, St. Georges' Hospital (London, GB). Each sample was anonymised following national ethical laws.

\section{Candidate reference materials processing}

The raw materials used were three plasmapheresis materials from patients with high concentrations of PR3 ANCA IgG. The materials were collected at the Staten Serum Institute, (Copenhagen, DK) and coded as SSIA, SSIC and SSIG. Table 1 summarises the different candidate reference materials that were produced on the basis of these three materials. They include plasma serum based on the three raw materials, and processed serum from SSIA, and purified PR3 IgG ANCA spiked into processed or unprocessed serum from healthy donors, all types of materials both liquid frozen and freeze-dried. The processing of the material chosen to prepare the CRM from is described in detail elsewhere [12].

\section{Commutability studies}

Commutability studies were performed prior to the decision on the final format of the reference material and the production of the CRM. 
Table 1: Candidate reference material formats.

\begin{tabular}{|c|c|}
\hline \multicolumn{2}{|l|}{ First study } \\
\hline \multicolumn{2}{|l|}{ Liquid frozen } \\
\hline IRMM-PR3-1 & SSIA plasma \\
\hline IRMM-PR3-2 & SSIA serum \\
\hline IRMM-PR3-3 & SSIA processed serum \\
\hline IRMM-PR3-4 & SSIC serum \\
\hline IRMM-PR3-5 & SSIG serum \\
\hline IRMM-PR3-6 & Anti-PR3 IgG spiked into processed serum \\
\hline IRMM-PR3-7 & $\begin{array}{l}\text { Anti-PR3 IgG spiked into unprocessed } \\
\text { serum from healthy donors }\end{array}$ \\
\hline IRMM-PR3-8 & $\begin{array}{l}\text { Anti-PR3 IgG spiked into unprocessed } \\
\text { serum from healthy donors }\end{array}$ \\
\hline IRMM-PR3-9 & $\begin{array}{l}\text { Anti-PR3 IgG spiked into unprocessed } \\
\text { serum plus additives }\end{array}$ \\
\hline \multicolumn{2}{|l|}{ Freeze dried } \\
\hline IRMM-PR3-1* & Plasma \\
\hline IRMM-PR3-2* & Serum \\
\hline IRMM-PR3-3* & Processed serum \\
\hline IRMM-PR3-4* & Serum \\
\hline IRMM-PR3-5* & SSIG serum \\
\hline IRMM-PR3-6* & Anti-PR3 IgG spiked into processed serum \\
\hline IRMM-PR3-7* & $\begin{array}{l}\text { Anti-PR3 IgG spiked into unprocessed } \\
\text { serum from healthy donors }\end{array}$ \\
\hline IRMM-PR3-8* & $\begin{array}{l}\text { Anti-PR3 IgG spiked into unprocessed } \\
\text { serum from healthy donors }\end{array}$ \\
\hline IRMM-PR3-9* & $\begin{array}{l}\text { Anti-PR3 IgG spiked into unprocessed } \\
\text { serum plus additives }\end{array}$ \\
\hline \multicolumn{2}{|l|}{ Second study } \\
\hline \multicolumn{2}{|l|}{ Liquid frozen } \\
\hline SSIA & Processed serum \\
\hline SSIC & Processed serum \\
\hline SSIG & Processed serum \\
\hline
\end{tabular}

*Freeze dried samples.

These studies are important so as to establish the conditions under which a CRM should be prepared in order to behave like a fresh patient sample in the various commercial assays.

Performance of the commutability studies: In the first commutability study 18 different formats of candidate RMs were tested (Table 1). These samples were analysed with eight ELISAs, which are mostly used in UK-NEQAS studies (Table 2). The formats of the candidate RMs tested varied from plasma samples, to serum and to purified PR3 ANCA IgG spiked into them. All ELISA measurements were performed on three plates per assay. On each of the three plates 21 of the clinical samples were measured in duplicate. The nine remaining clinical samples and the candidate RM samples were measured in duplicate in two of the three plates.

In the second commutability study, three liquid frozen candidate RMs were analysed (Table 1). It was decided to analyse them and dilutions thereof, in order to ensure that the RM concentrations were in the measurement interval of the assays. Those samples together with 30 clinical samples were measured in triplicate on two ELISA plates. Samples taken from different aliquots were also measured in triplicates with an automated Phadia 250 platform. For
Table 2: Immunoassays used in the commutability studies.

\begin{tabular}{lll}
\hline Manufacturer & Assay & Assay \\
\hline $\begin{array}{lll}\text { First study } \\
\text { Eurodiagnostica }\end{array}$ & Wieslab Direct PR3 IU & ELISA \\
Eurodiagnostica & Wieslab Capture PR3 & ELISA \\
Eurodiagnostica & DIASTAT PR3 & ELISA \\
Euroimmun & anti-PR3-hr-hn ELISA (IgG) & ELISA \\
Immunoconcept & RELISA ${ }^{\circledR}$ PR3-ANCA TEST & ELISA \\
INOVA & QUANTA LiteTM PR-3 IgG & ELISA \\
Orgentec & ORG 618 Anti-PR3 (cANCA) & ELISA \\
Phadia & Varelisa PR3 ANCA & ELISA \\
Second study & & \\
Phadia & EliA PR3 & FEIA \\
Orgentec & ORG 518 Anti-PR3 (cANCA) & ELISA \\
Eurodiagnostica & DIASTAT PR3 & ELISA \\
Euroimmun & anti-PR3-hr-hn ELISA (IgG) & ELISA \\
\hline
\end{tabular}

PR3, proteinase 3; ELISA, enzyme linked immunosorbent assay; $\mathrm{FEI}$, fluorescent enzyme immunoassay.

both studies all reconstitution and dilution volumes were gravimetrically controlled and the dilution levels were calculated from the masses and the densities rather than from the intended volumes. All measurements were performed at St. Georges' Hospital (London, UK).

Data analysis: Commutability was assessed by comparing the results for candidate reference materials with the results obtained for clinical samples (Analyse-it Software, Leeds, UK). For each sample the average values per plate were calculated and used for the analysis. First Pearson's correlation coefficient was calculated for the results of the clinical samples for all pairs of assays. For the pairs with a correlation coefficient higher than 0.75 a linear regression was applied and the $95 \%$ prediction interval calculated. A candidate reference material or dilution thereof was considered commutable when its results were within the $95 \%$ prediction interval of this regression performed on the results from clinical samples.

\section{Characterisation of the certified reference material ERM-DA483/IFCC}

Homogeneity and stability of the CRM: For the assessment of the between-unit homogeneity of the final material a number of vials are selected corresponding approximately to the cubic root of the total number of vials produced. These vials are selected in a random stratified sampling scheme covering the whole batch. Triplicates were measured on the same day with a chemiluminescent immunoassay (QuantaFlash PR3). Regression analyses were performed to evaluate potential trends both in the filling sequence and in the analytical sequence.

Stability studies were performed according to an isochronous design [13]. In this approach, units are stored for a specified length of time at different temperatures whereupon the units are moved to conditions where further degradation is assumed to be negligible. At the end of the isochronous storage, the samples are analysed 
simultaneously under repeatability conditions. The data are analysed by calculating the regression line for protein mass concentration in relation to time, and determining whether the slope is significantly different from zero.

Assignment of a value for PR3 IgG ANCA in ERM-DA483/IFCC: A calibration solution was prepared from purified PR3 IgG ANCA and characterised to allow the value-assignment of ERM-DA483/IFCC. A value for the total IgG concentration in the calibrant was assigned using three routine methods selective for total IgG (turbidimetry or nephelometry) using ERM-DA470k/IFCC as the calibrant. The concentration of the PR3 IgG ANCA stock solution was $0.289 \mathrm{mg} / \mathrm{mL}$ (2.2\% uncertainty, 95\% confidence interval) [12].

The value assignment of ERM-DA483/IFCC was achieved using a value transfer protocol that can be considered as the reference procedure [14]. It consists of use of dilutions of the IgG PR3 ANCA calibration solution and routine IgG PR3 ANCA assays. The participating laboratories were selected based on the market share of the assays used, and on criteria that comprised technical competence and meeting the requirements of ISO/IEC 17025 [15] or ISO 15189 [16]. Each participant used their own platform and reagents and was provided with detailed sample treatment protocols. The techniques used were traditional ELISAs and in some cases their variations (chemiluminescent and fluorescence immunoassays) specific for PR3 ANCA IgG [12]. A list of the assays is presented in Table 3. The samples consisted of vials of the CRM and eight dilutions of purified PR3 ANCA IgG prepared at the facilities of JRC. The participating laboratories had to reconstitute the CRMs and prepare seven dilutions of each vial. Measurements of all dilutions (of the reference material and the PR3 IgG ANCA calibration solution) were performed in triplicate in a single analytical run on each of 4 days.

Laboratories reported their raw data to the JRC. These data were assessed for their compliance with the analysis protocol and for their validity based on solely technical issues. No data were disregarded based on statistical analysis alone. Linear regressions were plotted for most of the assays while for some others a non-linear regression was observed and were treated accordingly (Table 3). Transfer factors were calculated for each of the datasets from the ratio of the slopes (concentration versus dilution) obtained for dilutions of the CRM and of the calibrant, as well as the concentration of the purified PR3 ANCA $\operatorname{IgG}[12]$

Table 3: List of immunoassays used in the characterisation study.

\begin{tabular}{lrll}
\hline Laboratory & $\begin{array}{r}\text { Assay } \\
\text { number }\end{array}$ & Assay name & Regression \\
\hline L1 & 1 & $\begin{array}{l}\text { ImmuLisaTM Proteinase 3 } \\
\text { (PR3) antibody Enhanced }\end{array}$ & Linear \\
L2 & 2 & ORG 618 PR3 hs & Linear \\
L3 & 3 & BioPlex 2200 Vasculitis & Linear \\
& 4 & Anti-PR3 EIA & Linear \\
L4 & 5 & QUANTA Lite PR 3 & Non-linear \\
& 6 & QUANTA Flash & Linear \\
L5 & 7 & PR3 ANCA Wieslab & Linear \\
L6 & 8 & AESKULISA PR3 sensitive & Linear \\
L7 & 9 & EliA PR3 & Non-linear \\
L8 & 10 & Anti-PR3-hn-hr-ELISA (IgG) & Non-linear \\
\hline
\end{tabular}

\section{Results and discussion}

\section{Commutability}

\section{Assay performance}

The aim of the commutability experiments was to assess:

- The equivalence of results from different assays. This includes the systematic bias (slope of the regression lines when results from one assay are plotted against the results from another assay), and sample-specific effects due to different selectivities of the assays (scattered around the regression line)

- The equivalence of the reference materials with routine samples

In order to focus on these issues the repeatability and intermediate precision were evaluated and experiments were designed with a sufficient number of replicates so as to minimise their impact. Thus, the observed scatter when comparing results from different assays is predominantly due to sample-specific effects, and not assay repeatability, issues with calibration, etc. As selectivity of an immunoassay is meant to be the ability to measure the analyte of interest despite the presence of other constituents [17].

The coefficient of variation (CV) of averages within and between plates for all samples was calculated. These calculations were performed both for the absorbance signal produced by the assays and the concentrations calculated from these absorbances. The results from absorbances showed a low intra-plate variation for all ELISAs. The inter-plate variation was higher, but below $7 \%$ for all assays. When the absorbances were converted into concentrations the CVs were higher. Still, between-plate CVs were below 15\%.

The higher CVs for concentration values could be due to the fact that assays designed for the detection of PR3 ANCA IgG include measurement intervals on the flatter part of the response curves, where small signal changes can mean large concentration changes. Additionally, not all assays use the same number of calibration points. Occasionally the distribution of these points, especially when they are less than 5 is unbalanced, with fewer calibration points at the upper range of the measuring interval.

\section{Immunoassay response for dilutions of clinical samples}

In the second commutability study, dilutions of the chosen candidate CRMs were measured with four assays. Figure 1 shows the results of clinical samples, candidate CRMs 


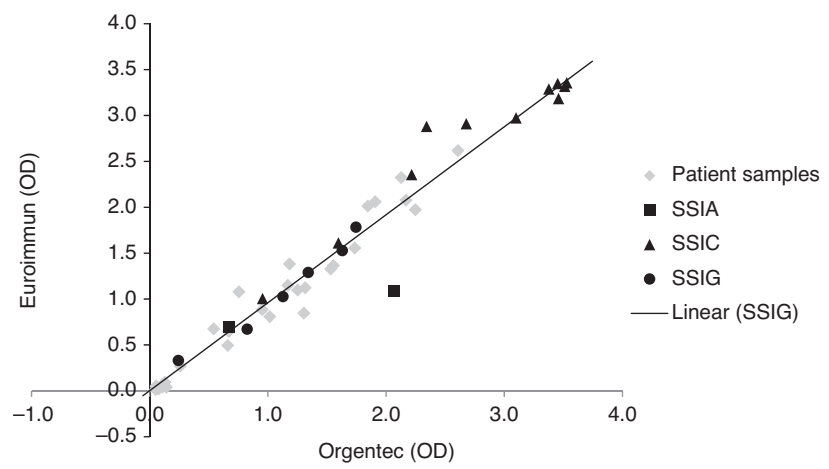

Figure 1: OD responses for the candidate CRMs SSIA (squares), SSIC (triangles) and SSIG (circles), dilutions thereof and patient samples (rhombus).

The values obtained in the case of SSIC are on the high end of the curve and above the limit of detection for the ELISAs used.

and dilutions thereof when analysed with an Euroimmun (Anti-PR3-hn-hr ELISA [IgG]) and an Orgentec kit (ORG 518). It can be observed that patient samples, SSIC, SSIG and their dilutions are commutable for the combination of these two methods. In the case of SSIA, it seems that one of the dilutions is not commutable. Based on these data, SSIG was eventually selected for preparing the CRM.

\section{Correlation between results from different assays}

The degree of correlation of results from different assays was evaluated by a pairwise comparison of results from all assays. Pearson's correlation coefficient (r) was calculated and the result interpretation was based on clinical biostatistics rules [18] according to which $r$ values ranging from 0.75 to 1 point to a correlation which is good to excellent. Examples of good and bad commutability for the CRM are shown in Figure 2. Table 4 shows Pearson's correlation coefficient for all assay comparisons of the first commutability study. They varied from moderate-to-very high for all assays except from the couples including the Wieslab Capture assay, which has a different selectivity. For 14 assay pairs the value of $r$ was above 0.75 , indicating a very good correlation. Moreover, five of these comparisons had
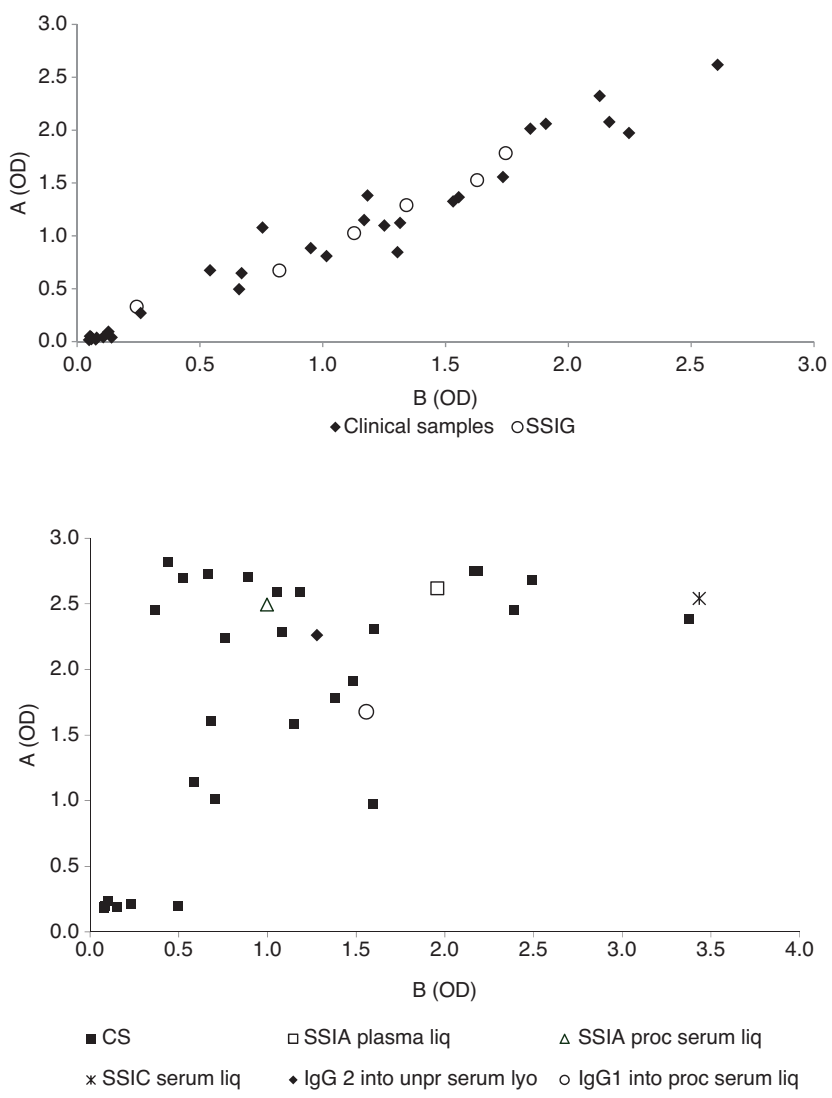

Figure 2: Example of results for the commutability study. Results for clinical samples are shown with a rhombus sign, results for SSIG as empty circles. The upper graph shows a case where the candidate RM is commutable. The lower graph shows an example where the candidate RMs is not commutable between the two methods compared.

CS, clinical sample; liq, liquid; proc, processed; unproc: unprocessed.

an $r$ value above 0.9 , indicating an excellent correlation of the measurement results. Despite the generally good picture of the results, some of the clinical samples were outliers in some of the comparisons.

\section{Classification and interpretation of measurement results}

The results for the clinical samples were classified as positive, inconclusive or negative by using the specific cut-off

Table 4: Pearson's correlation coefficient for the first commutability study.

\begin{tabular}{lcccrr}
\hline & Wieslab Direct PR3 IU & Euroimmun & QUANTA Lite PR 3 & ORG 618 PR3 hs & DIASTAT PR3 \\
\hline Varelisa PR3 & 0.88 & 0.80 & 0.91 & 0.98 & 0.74 \\
Wieslab Direct PR3 IU & & 0.85 & 0.97 & 0.94 & 0.83 \\
Euroimmun & & & 0.86 & 0.89 \\
QUANTA Lite PR 3 & & & & 0.96 \\
ORG 618 PR3 hs & & & & 0.77 \\
\hline
\end{tabular}


values of the different methods. In the first commutability study, 24 out of the 30 samples had the same classification among all the assays used. Nineteen samples were classified as being positive while six samples were found to be negative. The six remaining samples had different classifications in the different assays.

\section{Commutability of candidate reference materials}

The main purpose for performing the described commutability studies was to select the best material for the eventual development of a CRM. In the first commutability study several different formats of the candidate RM were tested. As the concentrations of the undiluted plasmapheresis materials were rather high compared to the patient samples a second, smaller scale, commutability study was used to make the final selection of the reference material format.

The results from the first commutability study of the candidate reference materials was monitored for all of the 28 combinations of assays for which Pearson's correlation coefficient was above 0.75 . In correlation coefficients below 0.75 , the prediction interval is very broad. This means that if the CRM is within this interval, it cannot be meaningful anymore.

Commutability can be analysed with different approaches as with a Passing-Bablok or Deming regression and evaluation of the results for the reference material with respect to the $95 \%$ prediction interval.

Overall, the commutability of the material SSIG, including its dilutions, is good. In Figure 3 pairwise comparisons of the clinical samples and the CRM are shown. This CRM is processed as the material ERM-DA470k/IFCC, which is certified for its total IgG content. This material is known to be stable for at least 10 years for IgG. Therefore, a similar stability is assumed for this new material. In addition, the material, as for any CRM produced by our Institute, is included in a yearly stability monitoring programme.

\section{Development of ERM-DA483/IFCC}

According to the current guidelines, in order for a reference material to be used as a calibrator, it has to fulfil a certain number of criteria: it has to be homogeneous between and within the values produced, it must be stable during transport and storage and must be commutable [10]. Additionally, as important is the possibility to assign a property value that is traceable to a stable reference.
ERM-DA483/IFCC was prepared as for previous materials [19]. From the performed commutability studies it was found that the conversion into serum, freeze-drying and the addition of preservatives did not interfere with the commutability of the material. SSIG plasmapheresis material was thus chosen for the development of the CRM, ERM-DA483/IFCC [12].

\section{Homogeneity and stability of the CRM}

\section{Homogeneity}

The determination of the between-vial heterogeneity is a key requirement for any CRM aliquoted into units [20]. The homogeneity study was performed by measuring 14 vials, each in triplicate. The standard deviation between vial $\left(s_{\mathrm{bv}, \mathrm{rel}}\right)$ and standard deviation within vial $\left(s_{\mathrm{wv}, \mathrm{rel}}\right)$ were determined by performing an ANOVA of the results. The standard deviation between batch $\left(s_{\mathrm{bv}, \mathrm{rel}}\right)$ is less than $1 \%$ (Table 5), and the material is thus sufficiently homogenous for its intended use.

\section{Stability}

Analysis of the CRM for PR3 showed that the material can be dispatched at temperatures of $-70^{\circ} \mathrm{C}$ or below, as the uncertainty associated with it for a period of 1 week was $0.1 \%$, which is negligible with respect to other uncertainties.

In regard to the long-term stability of the material, no statistical trends were observed at a $95 \%$ confidence level, neither after 6 nor after 12 months of storage. The associated uncertainty for a storage period of 12 months at $-70{ }^{\circ} \mathrm{C}$ was $1.14 \%$. Therefore the material should be stored at $-70{ }^{\circ} \mathrm{C}$.

\section{Value assignment}

For the assignment of a concentration value to the material, we followed the value transfer protocol as described by Blirup-Jensen et al. [14]. In the chosen approach, a value is transferred from a pure protein to the serum protein preparation. Based on the slopes plotted from the results between the purified PR3 ANCA IgG (pure protein) and the CRM (serum protein), the transfer factors for each assay are calculated and through them and through knowing the concentration of the PR3 ANCA IgG, it is possible to calculate the concentration of this protein in the CRM in 
A

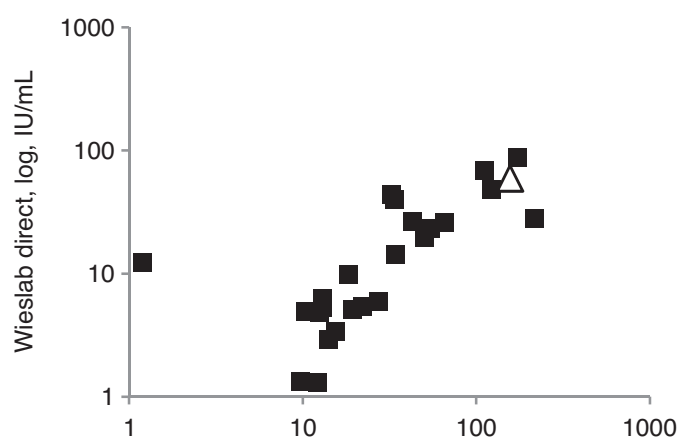

C

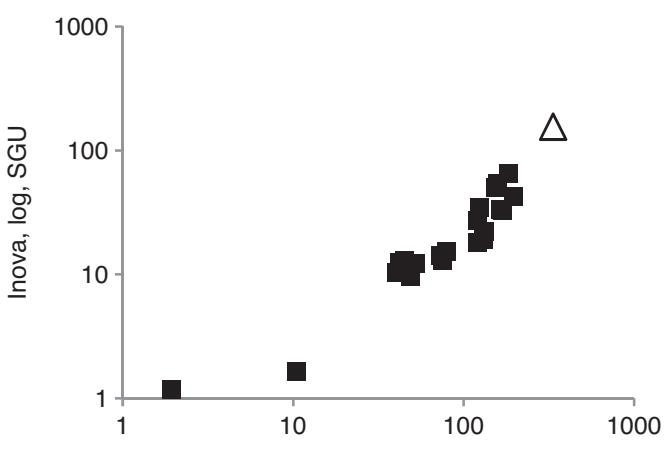

E

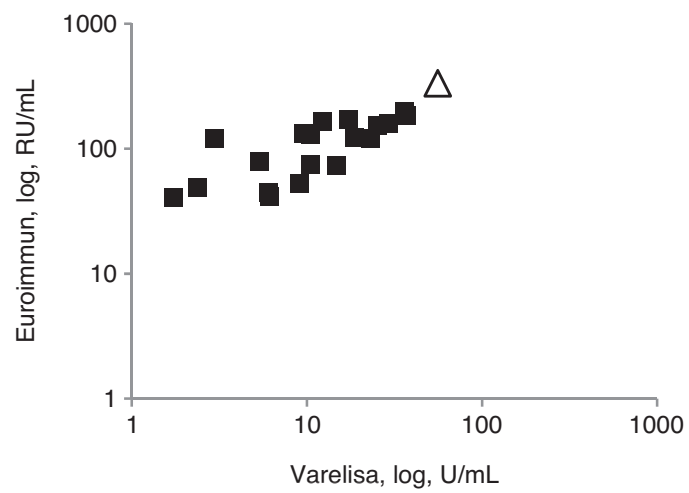

G

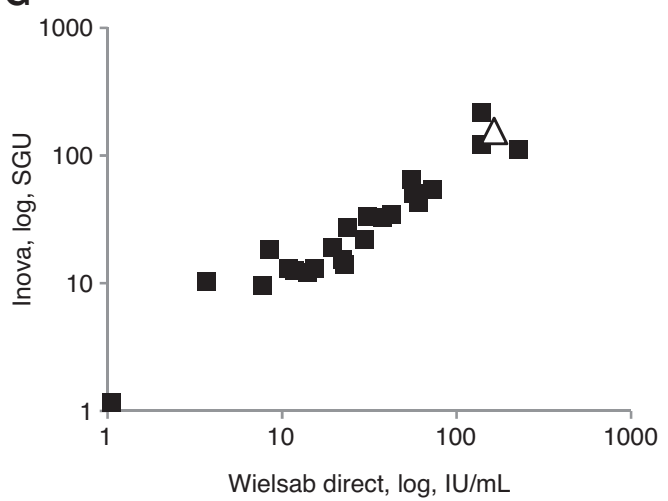

B

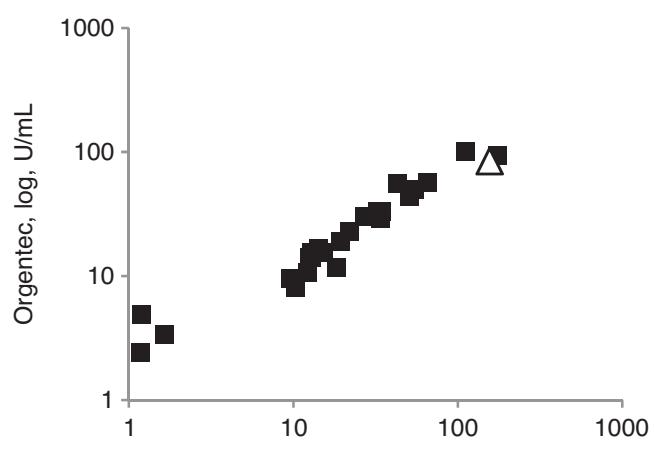

Inova, log, SGU

D

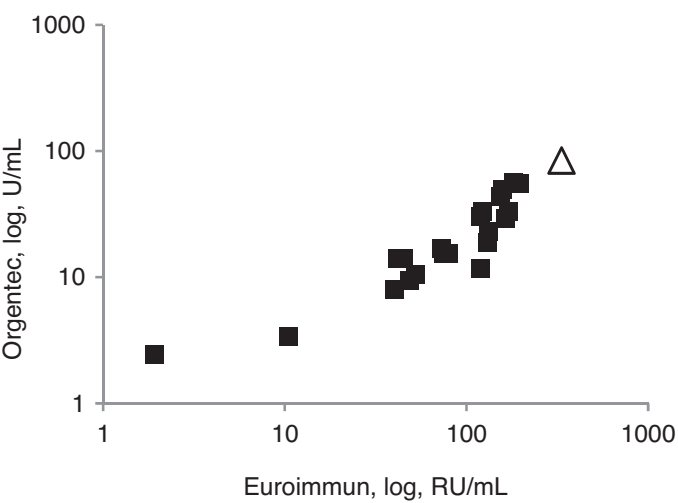

$\mathbf{F}$

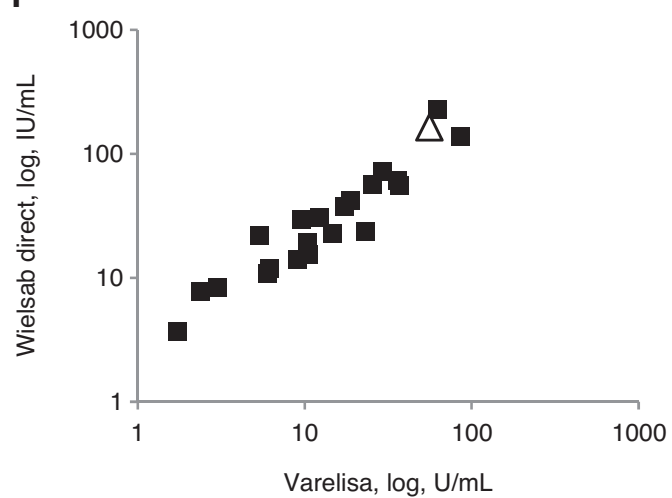

H

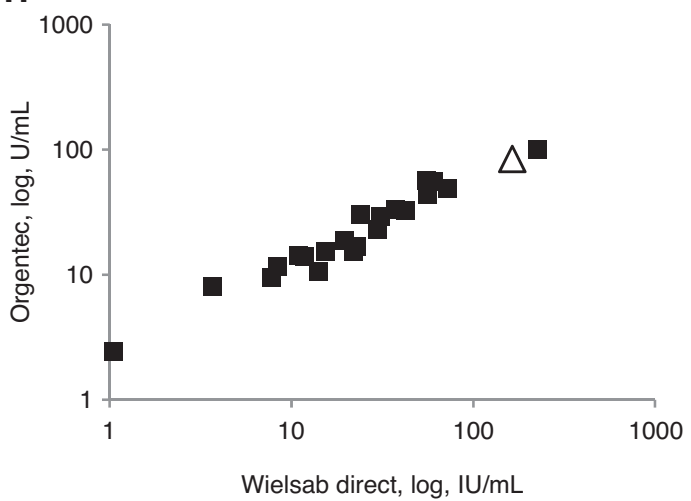

Figure 3: Examples of results for the commutability study.

In every graph from A-H, absorbance results for combinations of some of the assays used in the study are shown. Results for clinical samples are indicated with black squares and results for the selected CRM as empty triangles. The CRM is commutable in all cases even though not all curves are linear. 
Table 5: Uncertainty budget for the mass concentration of PR3 ANCA IgG in ERM-DA483/IFCC.

\begin{tabular}{lrrrrrrrr}
\hline CRM code & $\begin{array}{r}u_{\text {IgG PR3 ANCA rel }} \\
{[\%]}\end{array}$ & $\begin{array}{r}u_{\text {char rel }} \\
{[\%]}\end{array}$ & $\begin{array}{r}u_{\text {bb rel }} \\
{[\%]}\end{array}$ & $\begin{array}{r}u_{\text {sts rel }} \\
{[\%]}\end{array}$ & $\begin{array}{r}u_{\text {Its rel }} \\
{[\%]}\end{array}$ & $\begin{array}{r}u_{\text {CRM rel }} \\
{[\%]}\end{array}$ & $\begin{array}{r}\text { Certified value } \\
{[\mathrm{mg} / \mathrm{L}]}\end{array}$ & $\begin{array}{r}U_{\text {CRM }}(\mathrm{k}=2) \\
{[\mathrm{mg} / \mathrm{L}]}\end{array}$ \\
\hline ERM-DA483/IFCC & 2.75 & 4.37 & 0.97 & 0.09 & 1.14 & 5.33 & 270 \\
\hline
\end{tabular}

$u_{\mathrm{IgG} \mathrm{PR3} \mathrm{ANCA}}$, Standard uncertainty of the purified protein; $u_{\mathrm{char}}$, Standard uncertainty of the material characterisation; $u_{\mathrm{bb}}$, Standard uncertainty relating to a possible between-unit inhomogeneity; $u_{\text {sts }}$, Standard uncertainty of the short-term stability; $u_{\mathrm{tts}}$ Standard uncertainty of the long-term stability; $u_{\text {CRM }}$, Combined standard uncertainty of the certified value; $U_{\text {CRM }}$, Expanded uncertainty of the certified value.

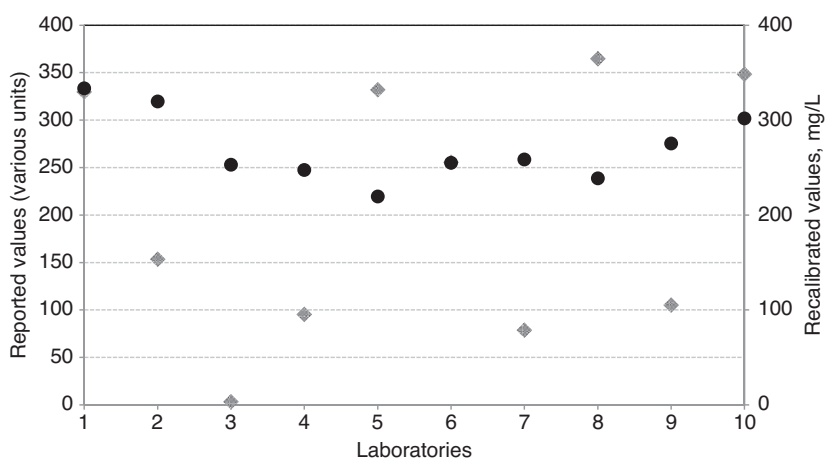

$\bullet$ Without common calibrant $\bullet$ With common calibrant

Figure 4: Comparison of response values for each laboratory participating in the study before and after correction with the CRM used as calibrant.

With the grey rhombus signs the values as reported by the laboratories are shown (in various units). With the black circles, those values were corrected using the CRM and are finally reported in the same unit $(\mathrm{mg} / \mathrm{L})$.

an SI unit (mg/L). The detailed procedure is published in the certification report of the material [12] and the analytical principles on which the characterisation measurements are based on, are listed by the Joint Committee for Traceability of Laboratory Medicine (JCTLM).

Figure 4 shows the average values for the CRM for each of the assays. The plot shows both the values in the assay scale obtained using the assay calibrator and the values after recalibration with the common calibration solutions from the purified PR3 IgG ANCA. This graph demonstrates that by the use of a common calibrant, results are less dispersed.

\section{Uncertainty}

For every value property in a CRM, an uncertainty is being calculated and reported (Table 5). The assigned combined uncertainty is the combination of all relevant uncertainty contributions; it is calculated according to ISO/IEC Guide 98-3 [21]. The greatest contribution is coming from the characterisation process of the CRM, while another

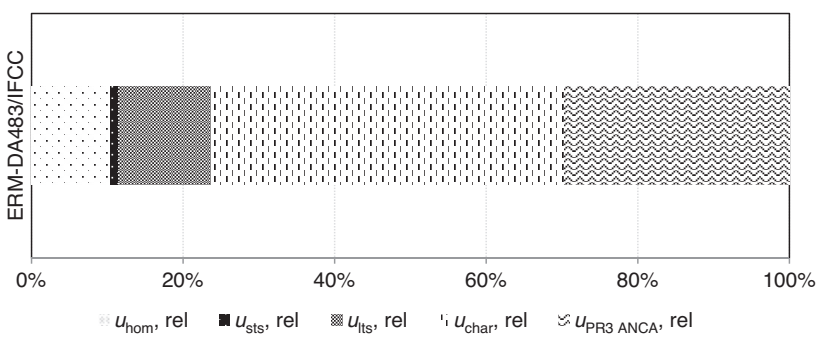

Figure 5: Graphic representation of the relative contribution of each parameter to the total uncertainty. Uncertainty related to homogeneity $\left(u_{\text {hom }}\right)$, short- $\left(u_{\mathrm{sts}}\right)$ - and long$\left(u_{\mathrm{lts}}\right)$ term stability, uncertainty related to the characterisation process of the CRM $\left(u_{\text {char }}\right)$ and of the purified protein $\left(u_{\text {PR3 ANCA }}\right)$.

important contributor is the characterisation of the purified PR3 ANCA IgG. Smaller contributors are the homogeneity and the long-term stability while a small part of the total uncertainty is due to the short-term stability of the CRM (Figure 5).

\section{Conclusions}

The impact of the lack of standardisation on laboratory results for ANCA has already been discussed [22]. A laboratory result needs to be accurate and conclusive so as to be of value to the requesting clinician ensuring correct diagnosis, prognosis and monitoring of the disease. Despite the evolution of testing and the automation of techniques and of their steps, laboratory results may still vary significantly and the assumption that the results are the same independently of the assay used is often incorrect.

Hutu et al. showed in a commutability study for MPO ANCA IgG that only 10 out the 30 clinical samples tested had the same classification amongst the assays used [23]. In our study for PR3 ANCA IgG antibodies the results were better, 24 out of 30 samples had the same classification. Nevertheless, it is worrying that a significant fraction of the assay results could lead to different conclusions in regard to the patient status if only the assay results would be taken into account. 
The results of these studies confirm that there is a considerable variability in results from PR3 ANCA IgG testing, despite the fact that the different assays performed well in terms of precision. We have performed two commutability studies in order to assess which PR3 ANCA IgG formats are commutable. The chosen format behaved in a manner comparable to the majority of typical samples in terms of inter-assay properties and dilution behaviour. Its use for calibration could reduce between-assay variation [23, 24], improve linearity between assay results with respect to the reference material and to each other, as demonstrated from the commutability data presented in this paper but also for the ERM-DA476/IFCC material, which is certified for its MPO ANCA IgG content [23], and eventually control the variation caused by lot to lot differences of the used reagents, as future lots of the same assay would be prepared based on the same CRM [23]. Assay results cannot always be harmonised by simple linear corrections, as results from some assays have a non-linear relationship to the PR3 IgG ANCA concentration and to results from other assays. A number of groups has worked on comparing the performance of different ELISAs for ANCA antibodies and have all concluded that despite the sensitivity and specificity of the assays available, variation due to the biochemical properties of the samples and antibodies present, exist [25-27]. These differences were still present between some of the assays when an older RM developed by the International Union of Immunological Societies (IUIS)/Centers for Disease Control and Prevention (CDC) was used [27].

Acknowledgments: The authors are grateful for the provision of raw materials by the Statens Serum Institute from Copenhagen, and the support of Allan Wiik and Niels Rasmussen. Stefanie Trapmann is greatly acknowledged for her constructive comments.

Author contributions: Evanthia Monogioudi and Ingrid Zegers wrote the paper; Ingrid Zegers, Joanna Sheldon, Heinz Schimmel and Pier Luigi Meroni designed the study; Dana Petronela Hutu performed the first commutability study, Evanthia Monogioudi the second one, Evanthia Monogioudi and Ingrid Zegers analysed the data. All the authors have accepted responsibility for the entire content of this submitted manuscript and approved submission.

Research funding: None declared.

Employment or leadership: None declared.

Honorarium: None declared.

Competing interests: The funding organisation(s) played no role in the study design; in the collection, analysis, and interpretation of data; in the writing of the report; or in the decision to submit the report for publication.

\section{References}

1. van Der Woude FJ, Lobatto S, Permin H, Van Der Giessen M, Rasmussen N, Wiik A, et al. Autoantibodies against neutrophils and monocytes: tool for diagnosis and marker of disease activity in Wegener's granulomatosis. Lancet 1985;325:425-9.

2. Goldschmeding R, van der Schoot C, Eten Bokkel Huinink D, Hack CE, van den Ende ME, Kallenberg CG, et al. Wegener's granulomatosis autoantibodies identify a novel diisopropylfluorophosphate-binding protein in the lysosomes of normal human neutrophils. J Clin Inv 1989;84:1577-87.

3. Koldingsnes W, Nossent HC. Epidemiology of ANCA associated vasculitis. Nor epidem 2008;18:37-48.

4. Jennette JC, Falk RJ, Bacon PA, Basu N, Cid MC, Ferrario F, et al. 2012 Revised international chapel hill consensus conference nomenclature of vasculitides. Arthritis Rheum 2013;65:1-11.

5. Bossuyt X, Cohen Tervaert J-W, Arimura Y, Blockmans D, FloresSuárez LF, Guillevin L, et al. Revised 2017 international consensus on testing of ANCAs in granulomatosis with polyangiitis and microscopic polyangiitis. Nat Rev Rheum 2017;13:683.

6. Vesper H, Emons H, Gnezda M, Jain CP, Miller WG, Rej R, et al. C53-A - Characterization and qualification of commutable reference materials for laboratory medicine - Approved guideline. CLSI \& IFCC 2010;30.

7. Miller WG, Myers GL, Rej R. Why commutability matters. Clin Chem 2006;52:553-4.

8. Van Blerk M, Van Campenhout C, Bossuyt X, Duchateau J, Humbel R, Servais G, et al. Current practices in antinuclear antibody testing: results from the Belgian External Quality Assessment Scheme. Clin Chem Lab Med 2009;47:102-8.

9. Meroni PL, Biggioggero M, Pierangeli SS, Sheldon J, Zegers I, Borghi MO. Standardization of autoantibody testing: a paradigm for serology in rheumatic diseases. Nat Rev Rheum 2014;10:35-43.

10. Miller WG, Schimmel H, Rej R, Greenberg N, Ceriotti F, Burns $\mathrm{C}$, et al. IFCC Working group recommendations for assessing commutability part 1: general experimental design. Clin Chem 2018;64:447-54.

11. Monogioudi E, Martos G, Hutu Dana P, Schimmel H, Meroni Pier L, Sheldon J, et al. Standardization of autoimmune testing - is it feasible? Clin Chem Lab Med (CCLM) 2018;25:56:1734-42.

12. Monogioudi E, Hutu DP, Charoud-Got J, Sheldon J, Schimmel H, Trapmann S, et al. Certification report: The certification of anti-proteinase 3 immunoglobulin $\mathrm{G}$ in human serum $\mathrm{ERM}^{\circledR}{ }^{\circledR}$ DA483/IFCC. EUR 28537 EN ed. Belgium: Publications Office of the European Union, 2017.

13. Lamberty A, Schimmel H, Pauwels J. The study of the stability of reference materials by isochronous measurements. Fresenius J Anal Chem 1998;360:359-61.

14. Blirup-Jensen S, Johnson AM, Larsen M. Protein standardization IV: Value transfer procedure for the assignment of serum protein values from a reference preparation to a target material. Clin Chem Lab Med 2001;39:1110-22.

15. Standardization IOf. ISO/IEC 17025:2005. General requirements for the competence of testing and calibration laboratories. Geneva, Switzerland, 2005.

16. Standardization IOf. ISO 15189:2012. Medical laboratories Requirements for quality and competence. Geneva, Switzerland, 2012. 
17. DeSilva B, Smith W, Weiner R, Kelley M, Smolec J, Lee B, et al. Recommendations for the bioanalytical method validation of ligand-binding assays to support pharmacokinetic assessments of macromolecules. Pharm Res 2003;20:1885-900.

18. Dawson B, Trapp RG. Research Questions About relationships among variables. In: Dawson B, Trapp RG, editors. Basic and Clinical Biostatistics. 4th ed.New York, NY: McGraw-Hill Companies, Inc, 2004.

19. Monogioudi E, Hutu DP, Martos Sevilla G, Tuddenham E, Sheldon J, Schimmel H, et al. Certification report: The certification of anti-myeloperoxidase immunoglobulin $\mathrm{G}$ in human serum ERM ${ }^{\circledR}$ - DA476/IFCC. EUR 27092 ed. Belgium: Publications Office of the European Union, 2015.

20. Standardization IOf. ISO 17034:2016 General requirements for the competence of reference material producers. Geneva, Switzerland, 2016.

21. Standardization IOf. ISO/IEC Guide 98-3:2008. Uncertainty of measurement - Part 3: Guide to the expression of uncertainty in measurement (GUM:1995). Geneva, Switzerland, 2008.

22. Monogioudi E, Hutu DP, Martos G, Sheldon J, Schimmel H, Meroni PL, et al. Development of a Certified Reference Material for myeloperoxidase-anti-neutrophil cytoplasmic autoantibodies (MPO-ANCA). Clin Chim Acta 2017;467:48-50.

23. Hutu DP, Tuddenham E, Monogioudi E, Meroni P, Schimmel H, Sheldon J, et al. First steps in the standardization of immunoglobulin IgG myeloperoxidase-anti-neutrophil cytoplasmic antibody measurements. Clin Exp Immunol 2016;183:193-205.

24. Merlini G, Blirup-Jensen S, Johnson AM, Sheldon J, Zegers I. Standardizing plasma protein measurements worldwide: a challenging enterprise. Clin Chem Lab Med 2010;48:1567-75.

25. Trevisin M, Pollock W, Dimech W, Melny J, Paspaliaris B, Gillis D, et al. Antigen-specific ANCA ELISAs have different sensitivities for active and treated vasculitis and for nonvasculitic disease. Am J Clin Pathol 2008;129:42-53.

26. Rasmussen N, Damoiseaux J, Csernok E, Heegaard Niels HH, Hellmich B, Paassen Pieter V, et al. Individual values of antineutrophil cytoplasmic antibodies do not correspond between antigen-specific assays. Clin Chem Lab Med 2018;56:e39-42.

27. Holle J, Herrmann K, Gross W, Csernok E. Comparative analysis of different commercial ELISA systems for the detection of antineutrophil cytoplasm antibodies in ANCA-associated vasculitides. Clin Exper Rheuma 2012;30(Suppl. 70):S66-9. 